\title{
Strain Coupling of a Nitrogen-Vacancy Center Spin to a Diamond Mechanical Oscillator
}

\author{
J. Teissier, A. Barfuss, P. Appel, E. Neu, and P. Maletinsky* \\ Department of Physics, University of Basel, Klingelbergstrasse 82, Basel CH-4056, Switzerland
}

(Received 7 March 2014; published 10 July 2014)

\begin{abstract}
We report on single electronic spins coupled to the motion of mechanical resonators by a novel mechanism based on crystal strain. Our device consists of single-crystal diamond cantilevers with embedded nitrogen-vacancy center spins. Using optically detected electron spin resonance, we determine the unknown spin-strain coupling constants and demonstrate that our system resides well within the resolved sideband regime. We realize coupling strengths exceeding $10 \mathrm{MHz}$ under mechanical driving and show that our system has the potential to reach strong coupling. Our novel hybrid system forms a resource for future experiments on spin-based cantilever cooling and coherent spin-oscillator coupling.
\end{abstract}

DOI: 10.1103/PhysRevLett.113.020503

Recent years have brought significant advances in the control of nanoscale mechanical oscillators, which culminated in experiments to prepare such oscillators close to their quantum ground state $[1,2]$ or a single-phonon excited state [3]. Generating and studying such states and further extending quantum control of macroscopic mechanical oscillators brings exciting perspectives for high precision sensing, quantum technologies [4], and fundamental studies of the quantum-to-classical crossover [5-7]. An attractive route towards these goals is to couple individual quantum two-level systems to mechanical oscillators and thereby enable efficient oscillator cooling [8] or state transfer [9] between a quantum system and oscillator in analogy to established concepts in ion trapping [10]. A prerequisite for most of these schemes $[8,10,11]$ is the resolved sideband regime, where the transition between the two quantum states exhibits well-resolved, frequency-modulated sidebands at the oscillator eigenfrequency. Various hybrid systems are currently being explored in this context and include mechanical oscillators coupled to cold atoms [12], superconducting qubits [3], quantum dots [13,14], or solid-state spin systems $[15,16]$. None of these systems, however, have reached the resolved sideband regime thus far and novel approaches are needed to further advance quantum control of macroscopic mechanical systems.

An important aspect that distinguishes existing hybrid systems is the physical mechanism they exploit to couple quantum system and oscillator. Coupling through electric [13], magnetic [15,16], or strain fields [14], as well as through optical forces [12] has been demonstrated as of now. Strain coupling is based on electronic level shifts $[8,17]$ induced by crystal strain during mechanical motion

Published by the American Physical Society under the terms of the Creative Commons Attribution 3.0 License. Further distribution of this work must maintain attribution to the author(s) and the published article's title, journal citation, and DOI.
PACS numbers: 03.67.Lx, 42.50.Wk, 76.30.Mi, 85.85.+j

and is particularly appealing in the context of hybrid systems. On one hand, strain coupling is predicted to result in interesting and unique system dynamics, such as spin squeezing [18] or phonon lasing [19] and can be used for mechanical spin driving [20]. On the other hand, strain coupling brings decisive technological advantages as it is intrinsic to the system. It thereby allows for monolithic and compact devices which are robust against manufacturing errors or thermal drifts and thus particularly amenable for future low-temperature operation. Despite these attractive perspectives, only few studies have exploited strain coupling for hybrid systems up to now [14]. While strain has previously been used to incoherently manipulate ensembles of spins in bulk diamond [20], resolved sideband operation or a quantitative determination of strain coupling constants remains an outstanding challenge as it requires the study of single spins in mechanical resonators of reduced dimensions.

In this Letter, we demonstrate for the first time the coupling of a mechanical resonator to an embedded single spin through lattice strain and present clear spectroscopic evidence that our system resides well within the resolved sideband regime. The devices we study consist of a single-crystal diamond cantilever with micron-scale dimensions, which contains an embedded single spin in the form of a negatively charged nitrogen-vacancy (NV) center [Fig. 1]. We achieve resolved sideband operation owing to the high mechanical frequency and sizable coupling strength in our structure. In addition, our room-temperature experiments yield the first quantitative determination of the previously unknown spinstrain coupling constants for the NV ground state.

Our diamond cantilevers consist of single-crystal, ultrapure diamond (Element Six) and were fabricated through recently developed top-down diamond nanofabrication techniques [22,23]. We fabricated our structures on the surface of (001)-oriented diamond starting material and aligned our cantilevers to within a few degrees to the [100] direction of the diamond [Fig. 1(a)]. Cantilever dimensions were in the range of $(10-50) \times 3.5 \times(0.2-1) \mu \mathrm{m}^{3}$ for 


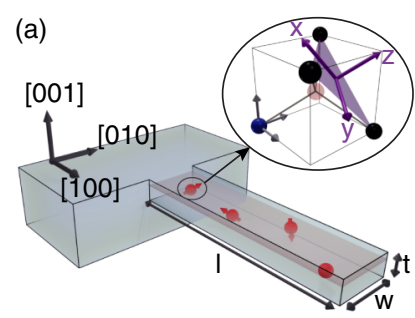

(b)

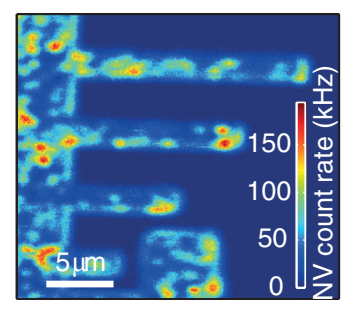

(c)

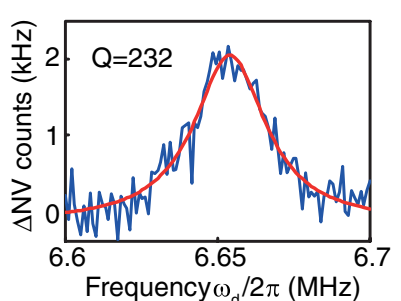

(d)

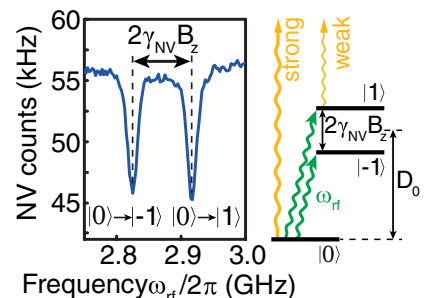

FIG. 1 (color online). (a) Schematics of the hybrid device studied in this work. Isolated electronic spins (red arrows) in the form of negatively charged nitrogen-vacancy (NV) centers (inset) are embedded in a diamond mechanical oscillator and coupled to the oscillator motion through crystal strain. (b) Confocal image of our cantilever devices showing individual, implanted NV centers scattered across the sample surface. (c) Mechanical excitation spectrum of a cantilever with typical resonance frequency and quality factor. The mechanical resonance was measured by monitoring the drop in NV fluorescence from the cantilever end, as the mechanical drive frequency $\omega_{d}$ was varied across $\omega_{\text {mech }}[21]$. (d) Optically detected electron spin resonance (ESR) of a NV center in a cantilever. The schematic illustrates the NV electronic ground-state spin configuration, which consists of a spin triplet, whose sublevels can be optically read out, since the $| \pm 1\rangle$ states yield less NV fluorescence than the $|0\rangle$ state.

length $l$, width $w$, and thickness $t$, respectively. The corresponding resonance frequencies $\omega_{\text {mech }}$ of the fundamental flexural mode of our cantilevers are estimated from Euler-Bernoulli thin beam theory and lie in a range of 1-10 MHz. A typical mechanical excitation spectrum [21] is shown in Fig. 1(c) and yields $\omega_{\text {mech }}=2 \pi \times 6.659 \mathrm{MHz}$, a linewidth of $\Delta \omega_{\text {mech }}=2 \pi \times 28.7 \mathrm{kHz}$, and a quality factor $Q=\omega_{\text {mech }} / \Delta \omega_{\text {mech }}=232$. The relatively modest value of $Q$ is caused by clamping losses and our experimental conditions under atmospheric pressure, and does not affect our experimental findings in any way.

The NV centers in our cantilevers were created through ${ }^{14} \mathrm{~N}$ ion implantation and subsequent sample annealing in vacuum at $800^{\circ} \mathrm{C}$. We chose an implantation density that allowed us to isolate single $\mathrm{NV}$ centers and an implantation energy resulting in an approximate NV depth of 10-15 nm from the cantilever surface. This depth forms a good compromise between NV spin coherence, and NV-cantilever strain coupling strength. We address the NV centers in our cantilever devices through a confocal microscope and show a typical confocal fluorescence image in Fig. 1(b). Individual NV centers are clearly visible as bright spots scattered throughout the device. We drive and detect NV spin transitions through a nearby microwave antenna and well-established [24] optical NV spin readout to perform optically detected electron spin resonance (ESR) [Fig. 1(d)].

In the following, we will focus on strain coupling of the NV's ground-state electronic spin sublevels to the motion of our diamond cantilevers. The NV ground state consists of a spin $S=1$ system with $S_{z}$ eigenstates $\{|-1\rangle,|0\rangle,|1\rangle\}$ and a zero-field splitting of $D_{0}=2.87 \mathrm{GHz}$ between $|0\rangle$ and $| \pm 1\rangle$ [Fig. 1(d)]. The degeneracy of $| \pm 1\rangle$ is lifted if the NV experiences magnetic or strain fields and the ground-state spin manifold can be described by the Hamiltonian [17,25]

$$
H / h=\underbrace{D_{0} S_{z}^{2}}_{H_{0}}+\underbrace{\gamma_{\mathrm{NV}} \vec{S} \cdot \vec{B}}_{H_{\text {Zeeman }}}+\underbrace{\tilde{d}_{\|} \epsilon_{z} S_{z}^{2}-\tilde{d}_{\perp} / 2\left[\epsilon_{+} S_{+}^{2}+\epsilon_{-} S_{-}^{2}\right]}_{H_{\text {strain }}},
$$

where $\gamma_{\mathrm{NV}}=2.799 \mathrm{MHz} / \mathrm{G}, h, \vec{B}$, and $\vec{S}$ are the NV gyromagnetic ratio, Planck's constant, the external magnetic field, and the NV electron spin operator (with components $S_{x}, S_{y}$, and $S_{z}$ and with $S_{ \pm}=S_{x} \pm i S_{y}$ ), respectively. The Hamiltonian $H_{\text {strain }}$ describes the coupling of the NV spin to lattice strain $\epsilon_{j}$ along coordinate $j$ $\left[j \in\{x, y, z\}\right.$ as defined in Fig. 1(a)] and $\epsilon_{ \pm}=-\epsilon_{y} \mp i \epsilon_{x}$. The strain coupling constants corresponding to strain longitudinal and transverse to the $\mathrm{NV}$ axis are denoted by $\tilde{d}_{\|}$and $\tilde{d}_{\perp}$. The effect of $\epsilon_{z}$ is equivalent to a modification of $D_{0}$ [26] and, therefore, only affects the energy difference between the states $| \pm 1\rangle$ and $|0\rangle$ without mixing any of the zero-field eigenstates. Conversely, if $\epsilon_{x, y} \neq 0,|1\rangle$ and $|-1\rangle$ mix and evolve into new eigenstates $|\tilde{1}\rangle$ and $|-\tilde{1}\rangle$. In the limit $\tilde{d}_{\perp} \epsilon_{x, y} \gg \gamma_{\mathrm{NV}}|\vec{B}|$, the energy difference between $| \pm \tilde{1}\rangle$ increases linearly with $\epsilon_{x, y}$ and $| \pm \tilde{1}\rangle=(|1\rangle \pm|-1\rangle) / \sqrt{2}$ [Figs. 2(a) and 2(b)].

In order to experimentally determine the unknown coupling constants $\tilde{d}_{\|}$and $\tilde{d}_{\perp}$, we applied variable degrees of strain to a NV center close to the clamping point of a cantilever by controlled cantilever bending. To that end, we employed a tungsten tip (Omniprobe, Autoprobe 250) mounted on a piezoelectric actuator and positioned this tip on the nonclamped end of the cantilever (with $t=1 \mu \mathrm{m}$ and $l=45 \mu \mathrm{m}$ ). We then displaced the tip to statically bend the cantilever, which in turn induced compressive or tensile lattice strain at the site of the NV [27]. We measured the effect of this strain on the NV by monitoring the optically detected ESR spectrum of the NV center as a function of the displacement $\delta$ of the cantilever's free end. Figure 2(c) shows the result of this experiment: As expected, the zerofield ESR line splits with cantilever displacement as a result of transverse strain. Additionally, a weak center-of-mass shift of the two resulting ESR lines is caused by $\epsilon_{z}$. We fitted the observed ESR line shifts by diagonalizing Hamiltonian (1) [white dashed lines in Fig. 2(c)] and obtained strain coupling constants $\tilde{d}_{\|}=5.46 \pm 0.31 \mathrm{GHz}$ and $\tilde{d}_{\perp}=19.63 \pm 0.40 \mathrm{GHz}$, where errors denote $95 \%$ 
(a)

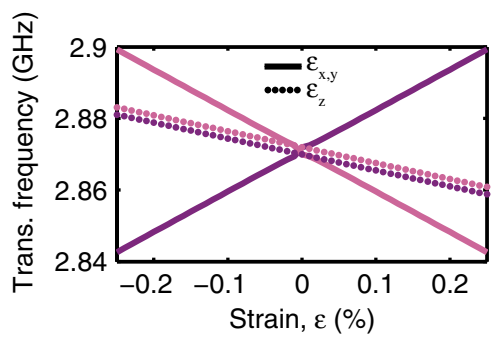

(b)

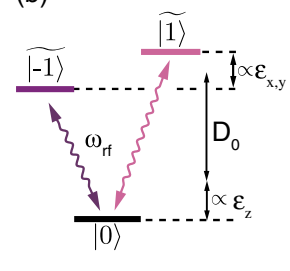

(c)

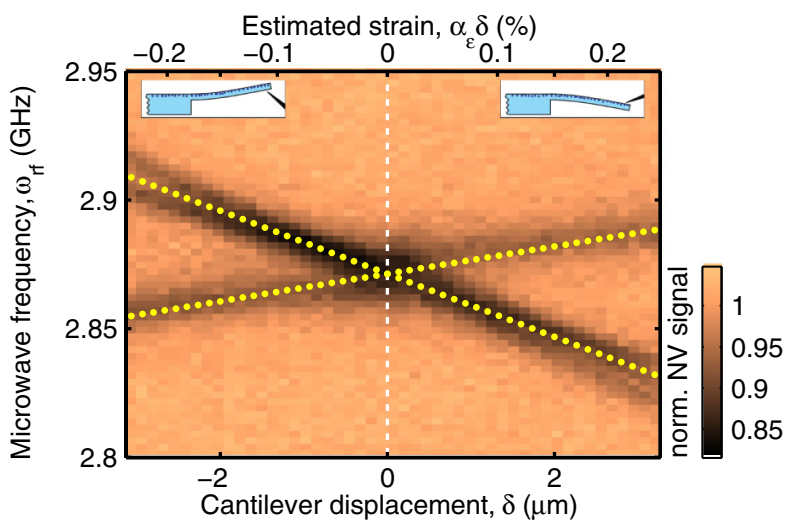

FIG. 2 (color online). (a) Calculated shifts of NV ESR transition frequencies as a function of crystal strain. The effects of strain transverse $\left(\epsilon_{x, y}\right)$ and longitudinal $\left(\epsilon_{z}\right)$ to the NV axis are plotted separately (solid lines and dotted lines, respectively). (b) Schematic of the NV electronic spin states and the action of $H_{\text {strain }}$. For $\epsilon_{x, y} \neq 0,|-\tilde{1}\rangle$ and $|\tilde{1}\rangle$ split, while for $\epsilon_{z} \neq 0,| \pm \tilde{1}\rangle$ shift with respect to $|0\rangle$. (c) Strain splitting of NV ESR lines as a function of static cantilever displacement. Positive and negative values of $\delta$ correspond to two different data sets (separated by the dashed line) and represent tensile and compressive strain at the $\mathrm{NV}$ location, respectively (see inset).

confidence intervals of our fits. For the fit, we assumed that the induced strain field at the cantilever surface $\epsilon_{[100]}$ is unidirectional and points along the direction of the cantilever (i.e., [100]), as expected from Euler-Bernoulli thin beam theory. Near the clamping point of the cantilever, we then find $\epsilon_{[100]}=(3 / 2)\left(t / l^{2}\right) \delta=\alpha_{\epsilon}^{[100]} \delta$, which for our cantilever yields $\alpha_{\epsilon}^{[100]}=7 \times 10^{-4} \mu \mathrm{m}^{-1}$. Within this approach, the ratio of $\epsilon_{z}$ to $\epsilon_{x, y}$ is constant and given by the orientation of the cantilever with respect to the NV axis, which in our case [Fig. 1(a)] yields $\epsilon_{z}=\sqrt{2 / 3} \epsilon_{[100]}$ and $\epsilon_{x, y}=\sqrt{1 / 3} \epsilon_{[100]}$ for all NVs.

Our determination of $\tilde{d}_{\|}$and $\tilde{d}_{\perp}$ is qualitatively consistent with theoretical expectations [21] and yields similar values on most NVs we studied. Interestingly, we also observed NVs whose ESR spectra showed significantly different behavior in response to beam bending, compared to the NV presented in Fig. 2 [21]. Both the magnitude of the measured strain shift per cantilever displacement and the ratio of transverse to longitudinal strain varied by up to 1 order of magnitude in some cases. While we expect $\tilde{d}_{\|, \perp}$ to be constant for all NVs, we assign these observations to variations in direction and magnitude of the local strain field at different NV sites for a given cantilever displacement. In particular, close to surfaces, strain fields are known to exhibit strong variations on the nanoscale [28] due to crystal imperfections and boundary effects. It is thus plausible that for some NVs the local strain field deviates strongly from expectations based on Euler-Bernoulli theory.

After we have established a significant coupling of NV spins to cantilever bending through static strain, we now turn our attention to the dynamics of our hybrid spinoscillator system. To that end, we provided a mechanical drive to the diamond cantilever by means of a piezoelectric transducer, placed in proximity to our sample and driven at a frequency $\omega_{d}$ with voltage $V_{\text {piezo }}$. We then characterized the resulting dynamical spin-cantilever interaction through high-resolution ESR spectroscopy [29]. For this experiment, we chose a NV where the induced strain field acts purely longitudinally. Additionally, we applied a magnetic field $B_{z}=26 \mathrm{G}$ along the $\mathrm{NV}$ axis, such that our discussion can be restricted to the two-level subspace spanned by $|0\rangle$ and $|-1\rangle$ and mixing of $|1\rangle$ and $|-1\rangle$ by transverse strain can be neglected. The cantilever drive can then be described by a classical phonon field, which leads to a timemodulated term $\tilde{d}_{\|} \epsilon_{z}^{\max } S_{z} \cos \left(\omega_{d} t\right)$ [30] in Hamiltonian (1) [31], where $\epsilon_{z}^{\max }=\alpha_{\epsilon}^{z} \delta^{\max }$ and $\delta^{\max }$ is the maximal cantilever amplitude. Using static beam bending, we measured a strain shift of $\alpha_{\tilde{\varepsilon}}^{z} \tilde{d}_{\|}=19 \mathrm{MHz}$ per micron of cantilever displacement [21] for the NV investigated here. The result of dynamic strain modulation can be seen in Fig. 3(a), where we choose $\omega_{d}=\omega_{\text {mech }}$ and compare highresolution NV ESR spectra of the $|0\rangle \rightarrow|-1\rangle$ transition in the presence and absence of the mechanical excitation. Without mechanical drive (upper trace), we observed the well-established hyperfine structure of the NV electron spin, which consists of three ESR lines split by the ${ }^{14} \mathrm{~N}$ hyperfine coupling constant $\omega_{\mathrm{hf}}=2 \pi \times 2.166 \mathrm{MHz}$ [32]. Upon resonant mechanical excitation, however, two clearly resolved, mechanically induced sidebands appear for each of the three hyperfine split ESR lines at detunings $\pm \omega_{\text {mech }}$, respectively. This experiment demonstrates that our system resides well within the resolved sideband regime of spinoscillator coupling, since the ESR linewidth $\Delta \omega<\omega_{\text {mech }}$ by a factor of $3(\Delta \omega=2 \pi \times 1.8 \mathrm{MHz})$.

To prove the resonant character of our optomechanical coupling and the mechanical origin of the observed sidebands, we extended the experiment presented in Fig. 3(a) by sweeping $\omega_{d}$ over a frequency range of $\pm 30 \mathrm{kHz}$ around $\omega_{\text {mech }}$, while monitoring the NV's ESR spectrum [Fig. 3(b)]. Clearly, sidebands only appear under resonant driving, when $\omega_{d} \approx \omega_{\text {mech }}$. Furthermore, the frequency range over which sidebands can be observed [Fig. 3(c)] closely matches $\Delta \omega_{\text {mech }}$, as determined from Fig. 1(c). This observation demonstrates that the observed sidebands are indeed induced by the mechanical oscillator and, in particular, excludes sidebands occurring through accidental modulation of the NV spin splitting by electric or magnetic stray fields. 
(a)

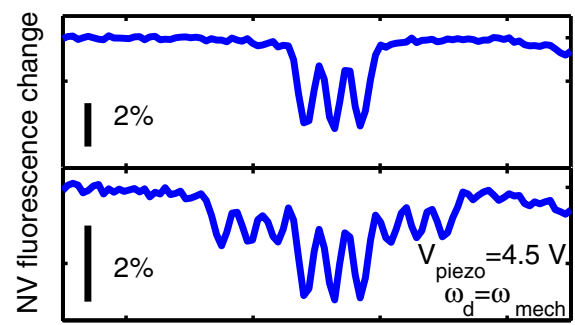

(b)

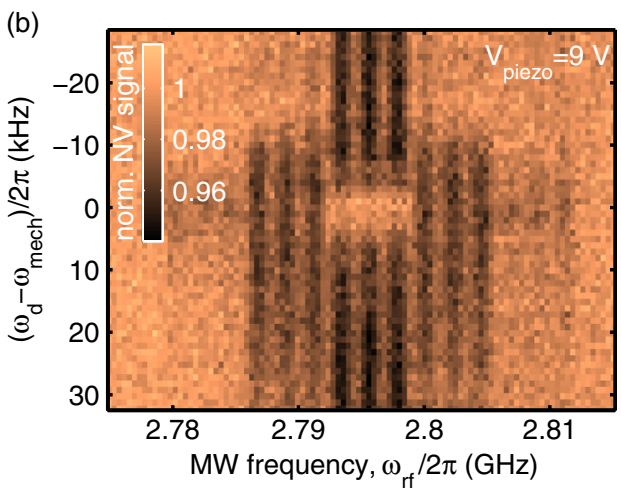

without mechanical drive

with mechanical drive

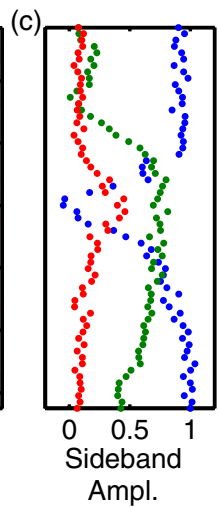

FIG. 3 (color online). (a) ESR trace of the NV in the cantilever in the absence (upper trace) and presence (lower trace) of resonant mechanical excitation. Without excitation, the ESR line exhibits a splitting into three hyperfine components. Mechanical driving induces sidebands to this central carrier at frequencies $\omega_{0} \pm \omega_{\text {mech }}$, where $\omega_{0}=2.796 \mathrm{GHz}$ is the bare ESR frequency of the NV center. (b) Evolution of the ESR sidebands as a function of mechanical drive frequency $\omega_{d}$ at an excitation amplitude $V_{\text {piezo }}=9 \mathrm{~V}$. A clear resonant behavior is observed with a maximal sideband amplitude appearing when $\omega_{d}=\omega_{\text {mech }}$. (c) Amplitude of the carrier signal (blue) and the two sidebands ( $n=1,2$ in green and red, respectively) as determined by a Lorentzian fit to the observed ESR dips. A slight asymmetry in the sideband amplitudes with respect to $\omega_{d}$ is caused by the onset of a mechanical nonlinearity of the diamond mechanical oscillator.

Finally, we investigate the evolution of the motioninduced sidebands as a function of the strength of the mechanical drive. Figure 4(a) shows a series of highresolution NV ESR traces recorded at various strengths of piezoexcitation with $\omega_{d}=\omega_{\text {mech }}$. For increasing $V_{\text {piezo }}$, we observe an increase of the sideband amplitude and eventually the appearance of higher-order sidebands up to order $n=3$. As expected [10], the amplitude of the $n$th sideband is well fitted by $J_{n}^{2}\left(\tilde{d}_{\|} \epsilon_{z}^{\max } / \omega_{\text {mech }}\right)$, where $J_{n}(x)$ is the $n$ thorder Bessel function of the first kind [see fits in Fig. 4(b)]. Next to a further confirmation of the nature of the sidebands, this measurement allows us to determine the strain coupling constant $\tilde{d}_{\|}$in this dynamical spin-strain coupling mode. We can extract the modulation depth $m=\tilde{d}_{\|} \epsilon_{z}^{\max } / \omega_{\text {mech }}$ as a function of drive amplitude and use an estimated mechanical susceptibility $\chi_{\text {mech }}=$ $\delta^{\max } / V_{\text {piezo }} \approx 23 \mathrm{~nm} / \mathrm{V}$ [21] of our system to relate $m$ to $V_{\text {piezo. }}$. This estimate yields $\alpha_{\epsilon}^{z} \tilde{d}_{\|} \approx 76 \mathrm{MHz} / \mu \mathrm{m}$ and lies within a factor of 4 of our earlier measurement of $\tilde{d}_{\|}$,

(a)

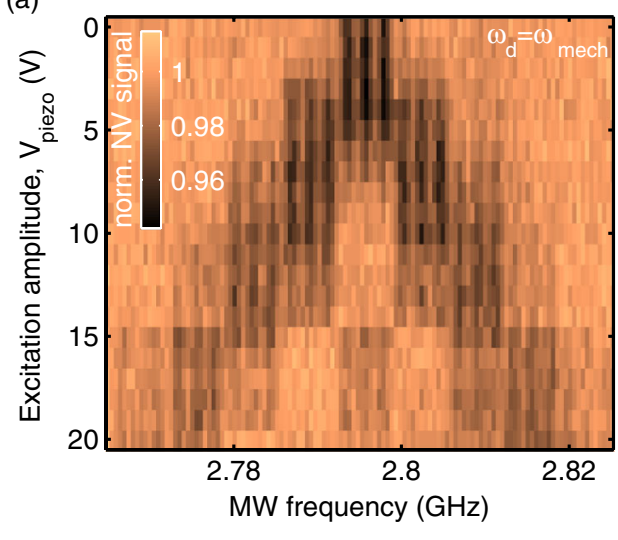

(b)

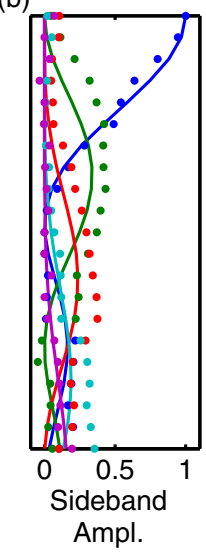

FIG. 4 (color online). (a) Amplitude of ESR sidebands for increasing excitation drive voltage $V_{\text {piezo }}$ of the mechanical oscillator. (b) Relative amplitudes of carrier signal (blue line) and sidebands as a function of excitation amplitude (dots). We determined the amplitudes by Lorentzian fit to the ESR peaks in (a) and normalized the dips in each line by the total measured ESR signal strength. Sidebands of order $n=1,2,3$ are color coded in red, green, and gray, respectively. Lines indicate squares of $n$ th-order Bessel functions.

which is reasonable given the uncertainty in our estimation of $\chi_{\text {mech }}$.

With spin-strain coupling and the resolved sideband regime clearly established, the question arises to what extent our system is amenable for future experiments in the quantum regime and, in particular, whether the strong coupling regime $\left(g_{0}^{2}>\gamma_{\text {mech }} \Gamma_{\mathrm{NV}}\right)$ can be achieved. The single-phonon coupling strength $g_{0}=\tilde{d}_{\|, \perp} \alpha_{\epsilon} x_{\mathrm{ZPM}}$ for our system is determined by the amount of strain per zero-point motion $x_{\mathrm{ZPM}}=\sqrt{\hbar /\left(2 m_{\mathrm{eff}} \omega_{\text {mech }}\right)}$ generated at the $\mathrm{NV}$ location and is largely determined by cantilever geometry $\left(g_{0} \propto \sqrt{1 /\left(l^{3} w\right)}\right)$. While for our cantilevers we find $g_{0} \approx 2 \pi \times 0.25 \mathrm{~Hz}$, an increase of $g_{0}$ to the kilohertz range [18] is within reach by further reducing dimensions of the mechanical oscillator [33]. $g_{0}$ has to be benchmarked against both the $\mathrm{NV}$ spin dephasing rate $\Gamma_{\mathrm{NV}}=2 \pi / T_{2}$ (with $T_{2}$ the NV spin coherence time) as well as the oscillator's thermal decoherence rate $\gamma_{\text {mech }}=k_{B} T / \hbar Q$ (with $k_{B}=21 \mathrm{GHz} / \mathrm{K}$ the Boltzmann constant and $T$ the bath temperature). With NV $T_{2}$ times approaching $1 \mathrm{sec}$ at low temperatures [34] and a projected value of $\gamma_{\text {mech }}=2 \pi \times 1 \mathrm{kHz}$ for $T=100 \mathrm{mK}$ and $Q=10^{6}$ [23], the strong coupling regime thus appears realizable in our system. The variations of strain coupling constants we observed additionally suggest that strain engineering in our devices could be employed to increase $g_{0}$ even further.

In summary, we have established NV centers embedded in single-crystal diamond nanomechanical resonators as a valuable resource for future experiments with hybrid systems in the quantum regime. In particular, we have firmly established the resolved sideband regime and quantitatively determined the NV-oscillator coupling strength. 
The nontrivial form of the spin-strain coupling Hamiltonian opens opportunities for exploring highly interesting avenues such as spin-induced oscillator sideband cooling [8], spin squeezing [18], or ultrafast, mechanical spin driving [20]. Finally, strain coupling of orbitally excited states is 5 orders of magnitude stronger $[35,36]$ compared to the values we established, which would bring our system deep into the ultrastrong coupling regime $\left(g_{0} \gg \omega_{\text {mech }}\right)$. Extending our experiments to cryogenic operation where coherent coupling to these states becomes accessible thus forms another highly exciting perspective.

We thank A. Högele. P. Treutlein, R. J. Warburton, S. D. Huber, and M. Pickova for fruitful discussions and valuable input. We gratefully acknowledge financial support through the NCCR QSIT, a competence center funded by the Swiss NSF, through the Swiss Nanoscience Institute, and through SNF Grant No. 200021_143697/1.

Note added.-We recently became aware of related work that determined NV spin-strain coupling constants [37]. The values reported there were obtained through coherent microwave spin-manipulation techniques and are consistent with our findings. The structures discussed in Ref. [37] do not allow for resolved sideband operation since there $\omega_{\text {mech }}<\Delta \omega$.

* Corresponding author. patrick.maletinsky@unibas.ch

[1] J. Chan, T. P. M. Alegre, A. H. Safavi-Naeini, J. T. Hill, A. Krause, S. Groblacher, M. Aspelmeyer, and O. Painter, Nature (London) 478, 89 (2011).

[2] J. D. Teufel, T. Donner, D. Li, J. W. Harlow, M. S. Allman, K. Cicak, A. J. Sirois, J. D. Whittaker, K. W. Lehnert, and R. W. Simmonds, Nature (London) 475, 359 (2011).

[3] A. D. O'Connell, M. Hofheinz, M. Ansmann, R. C. Bialczak, M. Lenander, E. Lucero, M. Neeley, D. Sank, H. Wang, M. Weides, J. Wenner, J. M. Martinis, and A. N. Cleland, Nature (London) 464, 697 (2010).

[4] P. Rabl, P. Cappellaro, M. V. Gurudev Dutt, L. Jiang, J. R. Maze, and M. D. Lukin, Phys. Rev. B 79, 041302 (2009).

[5] P. Treutlein, C. Genes, K. Hammerer, M. Poggio, and P. Rabl, arXiv:1210.4151.

[6] K. C. Schwab and M. L. Roukes, Phys. Today 58, 7, 36 (2005).

[7] W. Marshall, C. Simon, R. Penrose, and D. Bouwmeester, Phys. Rev. Lett. 91, 130401 (2003).

[8] I. Wilson-Rae, P. Zoller, and A. Imamoglu, Phys. Rev. Lett. 92, 075507 (2004).

[9] P. Rabl, S. J. Kolkowitz, F. H. L. Koppens, J. G. E. Harris, P. Zoller, and M. D. Lukin, Nat. Phys. 6, 602 (2010).

[10] D. Leibfried, R. Blatt, C. Monroe, and D. Wineland, Rev. Mod. Phys. 75, 281 (2003).

[11] P. Rabl, Phys. Rev. B 82, 165320 (2010).

[12] S. Camerer, M. Korppi, A. Jöckel, D. Hunger, T. W. Hänsch, and P. Treutlein, Phys. Rev. Lett. 107, 223001 (2011).

[13] S. D. Bennett, L. Cockins, Y. Miyahara, P. Grütter, and A. A. Clerk, Phys. Rev. Lett. 104, 017203 (2010).
[14] I. Yeo, P.-L. de Assis, A. Gloppe, E. Dupont-Ferrier, P. Verlot, N. S. Malik, E. Dupuy, J. Claudon, J.-M. Gérard, A. Auffèves, G. Nogues, S. Seidelin, J.-P. Poizat, O. Arcizet, and M. Richard, Nat. Nanotechnol. 9, 106 (2013).

[15] O. Arcizet, V. Jacques, A. Siria, P. Poncharal, P. Vincent, and S. Seidelin, Nat. Phys. 7, 879 (2011).

[16] S. Kolkowitz, A. C. Bleszynski Jayich, Q. P. Unterreithmeier, S. D. Bennett, P. Rabl, J. G. E. Harris, and M. D. Lukin, Science 335, 1603 (2012).

[17] J. R. Maze, A. Gali, E. Togan, Y. Chu, A. Trifonov, E. Kaxiras, and M. D. Lukin, New J. Phys. 13, 025025 (2011).

[18] S. D. Bennett, N. Y. Yao, J. Otterbach, P. Zoller, P. Rabl, and M. D. Lukin, Phys. Rev. Lett. 110, 156402 (2013).

[19] K. V. Kepesidis, S. D. Bennett, S. Portolan, M. D. Lukin, and P. Rabl, Phys. Rev. B 88, 064105 (2013).

[20] E. R. MacQuarrie, T. A. Gosavi, N. R. Jungwirth, S. A. Bhave, and G. D. Fuchs, Phys. Rev. Lett. 111, 227602 (2013).

[21] See Supplemental Material at http://link.aps.org/ supplemental/10.1103/PhysRevLett.113.020503 for additional information.

[22] P. Maletinsky, S. Hong, M. S. Grinolds, B. Hausmann, M. D. Lukin, R. L. Walsworth, M. Loncar, and A. Yacoby, Nat. Nanotechnol. 7, 320 (2012).

[23] P. Ovartchaiyapong, L. M. A. Pascal, B. A. Myers, P. Lauria, and A. C. Bleszynski Jayich, Appl. Phys. Lett. 101, 163505 (2012).

[24] A. Gruber, A. Drabenstedt, C. Tietz, L. Fleury, J. Wrachtrup, and C. Borczyskowski, Science 276, 2012 (1997).

[25] F. Dolde, H. Fedder, M. W. Doherty, T. Nöbauer, F. Rempp, G. Balasubramanian, T. Wolf, F. Reinhard, L. C. L. Hollenberg, F. Jelezko, and J. Wrachtrup, Nat. Phys. 7, 459 (2011).

[26] K. Fang, V. M. Acosta, C. Santori, Z. Huang, K. M. Itoh, H. Watanabe, S. Shikata, and R. G. Beausoleil, Phys. Rev. Lett. 110, 130802 (2013).

[27] Note that the spring constant of the manipulator was orders of magnitude higher than for the diamond cantilever, allowing us to directly relate the applied piezodisplacement to the induced cantilever bending amplitude.

[28] A. Béché, J. Rouvière, J. Barnes, and D. Cooper, Ultramicroscopy 131, 10 (2013).

[29] A. Dréau, M. Lesik, L. Rondin, P. Spinicelli, O. Arcizet, J. F. Roch, and V. Jacques, Phys. Rev. B 84, 195204 (2011).

[30] S. Rohr, E. Dupont-Ferrier, B. Pigeau, P. Verlot, V. Jacques, and O. Arcizet, Phys. Rev. Lett. 112, 010502 (2014).

[31] We note that in this limit our model is equivalent to spinoscillator systems coupled through magnetic field gradients $[4,15,16]$.

[32] X.-F. He, N. B. Manson, and P. T. H. Fisk, Phys. Rev. B 47, 8816 (1993).

[33] T. M. Babinec, B. J. M. Hausmann, M. Khan, Y. Zhang, J. R. Maze, P. R. Hemmer, and M. Loncar, Nat. Nanotechnol. 5, 195 (2010).

[34] N. Bar-Gill, L. M. Pham, A. Jarmola, D. Budker, and R. L. Walsworth, Nat. Commun. 4, 1743 (2013).

[35] A. Batalov, V. Jacques, F. Kaiser, P. Siyushev, P. Neumann, L. J. Rogers, R. L. McMurtrie, N. B. Manson, F. Jelezko, and J. Wrachtrup, Phys. Rev. Lett. 102, 195506 (2009).

[36] E. Togan, Y. Chu, A. Imamoglu, and M. D. Lukin, Nature (London) 478, 497 (2011).

[37] P. Ovartchaiyapong, K. W. Lee, B. A. Myers, and A. C. Bleszynski Jayich, arXiv:1403.4173. 